degenerative changes produced by agents of destructive nature. He does not, however, mention that reverse mutations back to, or towards, the normal are also produced by these means (Timoféeff-Ressovsky, $1934)^{4}$ : the agencies which inflict the wound in some instances, heal it in others ! Actually, such observations show us that mutations, normally occurring in many directions, are merely increased in frequency by heat and X-rays.

We may well suppose that, in the vast majority of instances, the type best fitted to natural conditions is that occurring in Nature. Furthermore, it is a commonplace of genetics that if domesticated strains are allowed to cross, the normal allelomorphs, separated by artificial selection, may be brought together, reproducing the wild-type condition. Simple Mendelian control of flower colour in Calceolaria, and natural selection favouring the wild-type, are all that is needed to explain the interesting instance of "reversion" quoted by Prof. MacBride.

Department of Comparative

E. B. FORD, Anatomy, Oxford.

1 W. E. Castle, Carnegie Inst. Wash., Publ. No. 320 (1922). 2 J. B. S. Haldane, "The Causes of Evvolution" (London, 1932).

ง W. Bowater, J. Gen., 3, 299-315 (1914).

\section{Evidence for Linear Units within Protoplasm}

IN his contribution to the "First Report on Viscosity and Plasticity", H. J. Jordan' ${ }^{1}$ has quoted only the measurements by W. Seifriz on protoplasm in 1924. But L. V. Heilbrunn does not find any conclusive evidence for elastic behaviour, apart from those cases where definite fibrillar elements can be observed in the protoplasm. In some recent papers, I have demonstrated the anomalous flow of protoplasm $^{2}$ and determined certain values interpreted as moduli of dilatability, transversal contraction and gliding ${ }^{3}$. Both the anomalous behaviour of protoplasm under varied shearing stresses and its elasticity are dependent on a structural organization of protoplasm not with respect to its grosser parts such as the nucleus, but in regard to the actual chemical molecules of which it consists ${ }^{4}$. So the next task was to find any substantial evidence for fibrillar or linear units within the protoplasm submitted to a stress along a definite axis.

My deformation apparatus ${ }^{5}$ consists of a capillary, a water manometer, and a tube with an india-rubber aspirator bulb, the three being united by a $Y$-tube. The protoplasm is sucked up into the capillary. At right angles to the axis of the capillary, a beam of parallel, plane polarized light passes with its optic axis at a given radius through the flowing protoplasm. After crossing the space below the objective, the light passes through the microscope and the analysing nicol. As the light is rendered telecentric by two diaphragms, only beams which run parallel, or almost parallel, to the axis of the microscope can enter the ocular.

In the former experiments, I have used the naked cells obtained from the liquefying pericarps of berries, or other plant cells obtained by means of experimental denudation. With the behaviour of these I have compared cells present in saliva (resembling leucocytes) obtained from the human mouth. Now $I$ have likewise accumulated data using living eggs of freshwater molluses, such as Unio and Anodonta, which are useful on account of their frequency and large size. After being isolated from the branchiæ of adult animals, the eggs were immersed in the ovary liquid.

The phenomenon observed between crossed nicols agrees with the assumed structure of protoplasm. If we permit the protoplasm to flow through the capillary, a system of many-coloured interference veins appears parallel to the capillary direction. With increasing speed of flow the interference lines disappear. Near the capillary axis, the flowing speed is greater than near the wall, so the coloured strips here are unrecognizable. The interference veins disappear after the eggs are placed in distinct buffer solutions of increasing hydrogen ion concentrations. In interpreting the results of experiments with eggs of different $p \mathrm{H}$, it must be remembered that cells often act much like amphoteric colloids having an iso-electric point ${ }^{6}$.

Summing up the results, we cannot be sure that the results obtained are quantitative. To anyone who has surveyed the whole field, however, there can be no question but that in all types of protoplasm hitherto used, fibrils or linear units are really present, although only visible if the protoplasm is flowing through a capillary.

A detailed description of the experiments will be given in a paper being published next year?

Kolonial- u. Uebersee-Museum, Hans H. Pfeiffer.

\section{Bremen.}

Nov. 12

${ }^{1}$ H. J. Jordan, First Report on Viscosity and Plasticity. p. 214, 221 (A msterdam, Noord-Hollandsche Uitg.-Maatsch., 1935).

${ }^{2}$ H. H. Pfeiffer, Physics, 7, 302 (1936).

${ }^{3}$ Pfeiffer, Cytologia, 6, 329 (1935). Protoplasma, 26, 372 (1936). 4 W. Seifriz, Protoplasm, 246, 261. (New York and London: McGraw-Hill, 1936.$)$

H. Pfeiffer, Protoplasma, 23, 210 (1935)

H. Pfeiffer, Trans. Faraday Soc., 29, 822, 833 (1930).

'H. Pfeiffer, Festschr. K. Fujii, in the press (Tokyo, 1937).

\section{Mechanism of Chemical Reaction in the Electric Discharge}

IN view of the apparent renewal of interest in chemical reactions taking place under the influence of the electric discharge, I venture to put forward the following suggestions, which have not so far been published although frequently expressed at colloquia.

The close proportionality often found, for example, by Dr. Keith Brewer and his collaborators ${ }^{1}$ between rate of reaction and current flowing in the outer circuit, has led to theories that positive ions are themselves the reacting bodies, clusters being formed, especially where more than one molecule of reaction product is obtained for each positive ion. Now although mobility data leave no doubt that surrounding molecules can exert a retarding effect upon an ion, to produce an effect which may formally be expressed in terms of ion-clusters, there is complete lack of both spectroscopic and positive ray evidence as to the existence of the reasonably stable aggregates which the theory necessitates. Moreover, it is not likely that the chemical properties of $\mathrm{N}_{2}+$, for example, will be the same as those of $\mathrm{N}_{2}$, while a process such as :

$$
\mathrm{N}_{2}++\mathrm{O}_{2} \underset{e}{\longrightarrow} 2 \mathrm{NO}+325 \text { Cal. }
$$

is so highly exothermic as to be incapable of giving the large yields, referred to ion-concentration, which have been reported. This same argument holds for many other reactions, since the ionization potentials concerned are generally of the order of $15 \mathrm{v}$. 